\title{
Variabilidad espacial y temporal en la comunidad de cladóceros de la Ciénaga de Paredes (Santander, Colombia) a lo largo de un ciclo anual
}

\author{
María M. Barón-Rodríguez ${ }^{1}$, Rosa A. Gavilán-Díaz ${ }^{2}$ y John J. Ramírez Restrepo. 3,* \\ 1 Programa de Biología, Universidad Industrial de Santander. AA 678 Bucaramanga. mmercedes30@yahoo.com \\ 2 Laboratorio de Limnología. Escuela de Biología. Universidad Industrial de Santander. AA 678 Bucaramanga. \\ Colombia.rgavilan@uis.edu.co \\ 3 Grupo de Limnología Básica y Experimental. Instituto de Biología. Universidad de Antioquía, Colombia.
}

* Autor responsable de la correspondencia: jjram@matematicas.udea.edu.co

\begin{abstract}
RESUMEN
Variabilidad espacial y temporal en la comunidad de cladóceros de la Ciénaga de Paredes (Santander, Colombia) a lo largo de un ciclo anual

En la Ciénaga de Paredes $\left(73^{\circ} 45^{\prime}-73^{\circ} 49^{\prime} \mathrm{W}\right.$ y $\left.7^{\circ} 26^{\prime}-7^{\circ} 29^{\prime} \mathrm{N}\right)$, ubicada en el Departamento de Santander (Colombia), se determinó la composición, la variación espacial y temporal de la estructura de la comunidad de cladóceros, con base en arrastres verticales con malla de $68 \mu \mathrm{m}$, en ocho estaciones de muestreo en un ciclo anual (febrero de 1998 a enero de 1999). Para evaluar la estructura, se utilizaron los números de Hill (N0, N1 y N2) y la equidad. El soporte del muestreo fue calculado con los estimadores Chao 1 y 2. La existencia de diferencias significativas de los números de Hill, la equidad, la densidad numérica, la columna de agua, el pH, el OD, y la temperatura, entre campañas y entre estaciones, se realizó a través de un ANDEVA. Las especies y morfoespecies encontradas (31) poseen distribución tropical, subtropical y cosmopolita; pertenecen generalmente a cuerpos de agua temporales, llanuras de inundación o ciénagas. Las mayores abundancias fueron registradas para Moina minuta, Moina cf. micrura, Diaphanosoma brevireme y Ceriodaphnia cornuta, las cuales representaron el $81.9 \%$ del total de individuos colectados. Los resultados obtenidos por los estimadores de riqueza indican que si se aumentara el esfuerzo de muestreo con las técnicas utilizadas, no incrementaría el número de morfoespecies. Con respecto a la variación espacial de la estructura, la estación $\mathrm{V}$ presento mayor equidad, riqueza y diversidad, pero menor densidad numérica, esta condición muestra la diferencia de esta estación en comparación con las demás; su tendencia atípica es explicada ya que dicha estación se encuentra cerca del afluente principal de la Ciénaga (Quebrada La Gómez). En la variación temporal, la estructura de la comunidad de cladóceros cambió entre campañas de muestreo ya que la equidad y la riqueza presentaron diferencias significativas, que se evidencian en el cambio de la abundancia relativa de las morfoespecies, mas no en la abundancia de cladóceros. Esto es causado por las fluctuaciones de la precipitación y el alto de la columna de agua.
\end{abstract}

Key words: Neotropic, floodplains, zooplankton, swamp, cladocerans, marsh.

\begin{abstract}
Spatial and temporal variability in the cladoceran community of the Cienaga of Paredes (Santander, Colombia) along an annual cycle

In the Ciénaga de Paredes $\left(73^{\circ} 45^{\prime}-73^{\circ} 49^{\prime} \mathrm{W}\right.$ and $\left.7^{\circ} 26^{\prime}-7^{\circ} 29^{\prime} \mathrm{N}\right)$, located in the Department of Santander (Colombia), the composition, and the spatial and temporal variation of the cladoceran community structure was determined with samples taken with a 68 m vertical-hauled net, at eight sampling stations in an annual cycle (February 1998 to January 1999). To evaluate the structure, Hill numbers (NO, N1 and N2) and evenness were used. The sampling support was calculated with the Chao 1 and 2 estimators. The existence of significant differences for Hill numbers, evenness, numeric density, water column, $\mathrm{pH}, \mathrm{OD}$, and temperature among field trips and among stations, was analysed through an ANOVA. The species and morphospecies found (31), had a tropical, subtropical, and cosmopolitan distribution; belonging to temporary water bodies, floodplains or "ciénagas". The highest abundances were registered for Moina minuta, Moina cf. micrura, Diaphanosoma brevireme and Ceriodaphnia cornuta, which represented $81.9 \%$ of the total collected individuals. The results obtained with the richness estimates suggest that if the sampling effort were increased using the same techniques, the morphospecies' number would have not increased. With regard to the structure's spatial variation, the station $V$ showed higher evenness, richness, and diversity, but lower numeric density; this condition shows the difference between this station and the other ones;
\end{abstract}


its atypical trend is explained by this station being near to the main tributary of the "Ciénaga" ("Quebrada La Gómez"). Regarding the temporal variation, the cladocerans' community structure changed between field trips, since the evenness and the richness showed significant differences, reflected by the variation in the relative abundance of the morphospecies but not in the cladocerans' abundance. This was caused by the fluctuations in rainfall and water level.

Palabras clave: Neotrópico, planos inundables, zooplancton, pantano, cladóceros, ciénaga.

\section{INTRODUCCIÓN}

Las ciénagas son de especial interés ya que son consideradas por algunos autores como planos de inundación (Junk et al., 1989; Junk, 1996). Son sistemas acuáticos que se encuentran en el trópico, están afectados por fluctuaciones en el alto de la columna de agua y poseen mayor diversidad de organismos que en cuerpos de agua de zonas templadas (Collado et al., 1984; Fernando et al., 1990; Dumont, 1994). Los estudios existentes sobre estos cuerpos de agua y la comunidad zooplanctónica y en especial de los cladóceros, se han enfocado en la influencia del ciclo hidrológico sobre dicha comunidad (Hardy et al., 1984; Zoppi de Roa et al., 1985; Pinto-Coelho, 1987; Bohrer et al., 1988; Lansac Tôha et al., 1993; Boze1li, 1994), o en la asociación de las especies a la condición trófica (Sendacz et al., 1984; Sendacz \& Kubo, 1999; Sampaio et al., 2002).

Los cladóceros, a pesar de ser uno de los componentes principales del zooplancton dulceacuícola, ha sido objeto de escasos estudios en regiones tropicales y especialmente en Colombia. En Suramérica, por ejemplo, se encuentran investigaciones sobre las variaciones espaciotemporales de los cladóceros en cuerpos de agua brasileros (Bohrer et al., 1988); descripciones de especies, así como su distribución, en algunas regiones colombianas (Roessler, 1994 y 1996); estudios en las sabanas inundables y bajo Orinoco de Venezuela (Zoppi de Roa et al., 1985 y Rey \& Vásquez, 1988); e inventarios en los cladóceros del Perú (Valdivia, 1988) y en Brasil (Elmoor-Loureiro, 1998).

En el presente trabajo, derivado del de Gavilán-Díaz (2000), se compararon las fluctuacio- nes del nivel del agua, con la densidad numérica de la comunidad zooplanctónica en tres ciénagas del Magdalena Medio Santandereano (Paredes, Chucurí y Llanito) a lo largo de un ciclo hidrológico. En dicho estudio la Ciénaga de Paredes se destaca por presentar mejores condiciones físicas y químicas que las otras ciénagas, para el desarrollo de los cladóceros, grupo que presentó la mayor abundancia relativa.

Se pretende en esta investigación responder a la pregunta: ¿Varía espacialmente y temporalmente la estructura de la comunidad de los cladóceros presente en la ciénaga? Si la estructura de la comunidad de los cladóceros varía espacial y temporalmente se prevé que, exista heterogeneidad entre las ocho estaciones y entre las campañas de muestreo en la Ciénaga de Paredes. Por lo tanto, el objetivo del presente trabajo fue determinar a partir de datos obtenidos en ocho estaciones de muestreo y en un ciclo anual, la variación espacial y temporal en la estructura de de la comunidad de los cladóceros presente en la Ciénaga de Paredes.

\section{ÁREA DE ESTUDIO}

La Ciénaga de Paredes pertenece al Valle Medio de la Cuenca del Río Magdalena (García \& Dister, 1990). Está ubicada en el Departamento de Santander (Colombia, $73^{\circ} 45^{\prime}-73^{\circ} 49^{\prime} \mathrm{W}$ y $7^{\circ} 26^{\prime}$ $7^{\circ} 29^{\prime} \mathrm{N}$ ), entre los Municipios de Sabana de Torres y Puerto Wilches (Fig. 1). Su afluente principal es la Quebrada La Gómez, (Cormagdalena, 1999), se encuentra a una altitud de 75 m.s.n.m., el alto de su columna de agua está determinado por el régimen de lluvias, y según la clasificación propuesta por Arias (1985) la Ciénaga 


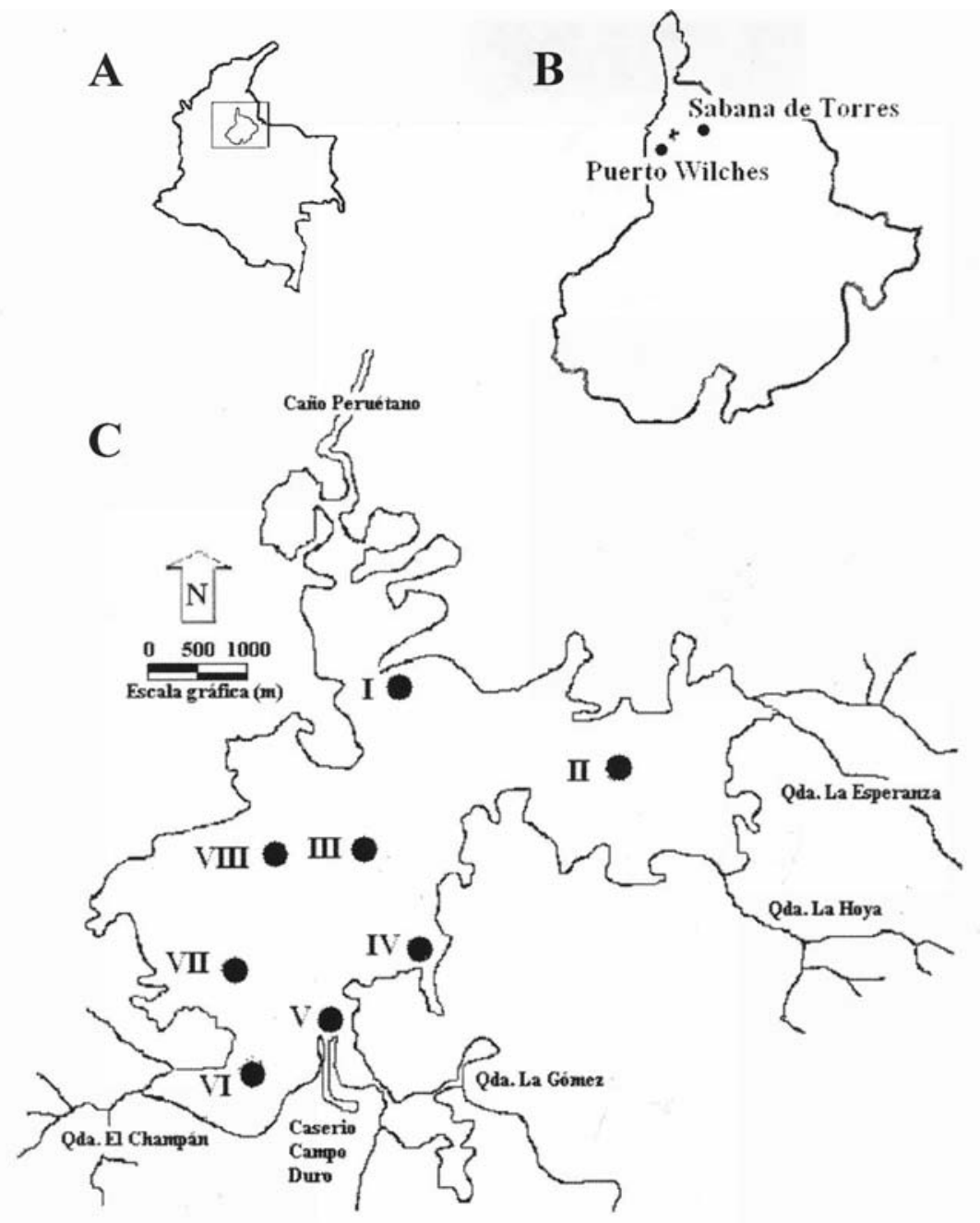

Figura 1. Localización y estaciones de muestreo: A. Colombia, B. Departamento de Santander y C. Ciénaga. Location and sampling stations: A. Colombia, B. Department of Santander and C. Ciénaga.

de Paredes es considerada como ciénaga tipo III, ya que se encuentra conectada al río Lebrija por el Caño Peruétano.

Según la clasificación de Holdrige (2000) la Ciénaga de Paredes es un cuerpo de agua que pertenece a un bosque seco Tropical (bs-T). La pluviosidad presenta un ciclo bimodal definido que alcanza los $3000 \mathrm{~mm}$ anuales, con valores máximos a finales de mayo y noviembre, y mínimos de diciembre a febrero (Arias, 1985; García \& Dister, 1990). Gavilán-Díaz (2000) registró temperaturas máximas de $38^{\circ} \mathrm{C}$; variación de oxígeno disuelto entre $10.8 \mathrm{mg} / \mathrm{l}$ y 0.53 $\mathrm{mg} / \mathrm{l}$; tendencia a la homogeneidad en la con- ductividad en la columna de agua, con valores máximos de $32.0 \mu \mathrm{S} / \mathrm{cm}$ y mínimos de $8.0 \mu \mathrm{S} / \mathrm{cm}$; y valores de $\mathrm{pH}$ entre 4.57 y 8.57 .

\section{MATERIALES Y MÉTODOS}

Los datos analizados fueron los obtenidos por Gavilán-Díaz (2000) en la Ciénaga de Paredes en ocho estaciones y en 11 campañas de muestreo (Febrero de 1998 a Enero de 1999). En la Tabla 1 y en la figura 1 se muestra la distribución de las estaciones en la ciénaga y las características físicas y químicas más importantes de la ciénaga 
Tabla 1. Característica principal, altura de la columna de agua, OD, temperatura y pH de las estaciones de muestreo en un ciclo anual en la Ciénaga de Paredes (Santander-Colombia). Main characteristic, water level, OD, temperature, and pH of the sampling stations in an annual cycle in the Paredes marsh (Santander-Colombia).

\begin{tabular}{|c|c|c|c|c|c|}
\hline Nombre Estación & Características & Columna agua (m) & OD (mg/l) & Temperatura $\left({ }^{\circ} \mathrm{C}\right)$ & pH \\
\hline I-Caño Peruétano & Efluente principal. & 2.1 & 7.13 & 31.3 & 6.72 \\
\hline II-Rincón de la Hoya & Afluente secundario & 1.8 & 6.61 & 31.9 & 6.93 \\
\hline III-Centro Ciénaga & Centro del cuerpo de agua. & 2.2 & 6.63 & 32.4 & 6.91 \\
\hline IV-Pozo Manatíes & Lugar más profundo & 2.6 & 6.46 & 32.8 & 6.41 \\
\hline V-Qda La Gómez & Afluente principal & 2.1 & 6.52 & 29.5 & 6.02 \\
\hline VI-Qda El Champán & Afluente secundario. & 1.8 & 6.25 & 32.9 & 6.56 \\
\hline VII-Frente a Campo Duro & Cerco de acentamientos humanos & 2.0 & 6.93 & 33.5 & 7.54 \\
\hline VIII-Casa Blanca & Cerca de asentamientos numanos. & 2.0 & 7.04 & 33.7 & 7.56 \\
\hline
\end{tabular}

en el tiempo de muestreo. Los perfiles del $\mathrm{pH}$, el OD y la temperatura se realizaron con un HORIBA Multiparámetro U-10. Las muestras biológicas fueron colectadas en cada campaña y cada estación de muestreo por medio de arrastres verticales a lo largo de la columna de agua, con una red de plancton de $30 \mathrm{~cm}$ de diámetro y $68 \mu \mathrm{m}$ de apertura de poro. Las muestras fueron fijadas con formol $4 \%$ y glucosa $8 \%$ (Haney \& Hall, 1973). La determinación de las especies de los cladóceros se efectuó, con base en claves taxonómicas (Brooks, 1959; Oliver, 1969; Pennak, 1978; Montú \& Goeden, 1986; Zambrano, 1989; Rey, 1991; Korovhinsky, 1992; Smirnov, 1992; Paggi, 1995; Lieder, 1996; Smirnov, 1996; Elmoor-Loureiro, 1997; Flössner, 2000; Korovchinsky \& Elías-Gutiérrez, 2000; OrlovaBienkowskaja, 2001) y la asesoría de personal especializado. Se realizó un análisis cualitativo con el fin de determinar las especies y morfoespecies presentes en cada una de las muestras; luego se cuantificaron en una cámara de recuento de $6 \mathrm{~cm} \times 6 \mathrm{~cm}$, en la cual se inicio contando $20 \mathrm{ml}$ de $100 \mathrm{ml}$ que contenían las muestras; si no se completaban las especies determinadas en el análisis cualitativo, se adicionaban $4 \mathrm{ml}$ de la muestra hasta completarlas (APHA, 1995; Wetzel \& Likens, 1990). El cálculo de los litros filtrados se realizó con base en la fórmula del volumen del cilindro en donde la altura y el diámetro del cilindro correspondían, respectivamente a la altura de la columna de agua en la estación de muestreo y al diámetro de la boca de la red de plancton. Las muestras biológicas forman parte de la Colección Limnológica del Museo de His- toria Natural de la Universidad Industrial de Santander (UIS ML 0013 a UIS ML 0187).

Se definió estructura como la composición, distribución y organización de la comunidad dentro de un área y un tiempo determinado, incluyendo sus interrelaciones y una de las maneras más simples de evaluarla es a través de la diversidad (Kempton, 1979; Margalef, 1991). Los evaluadores de estructura se calcularon con base en los números de Hill (N0, N1 y N2) (Hill, 1973) los cuales miden el grado en el que las abundancias son proporcionalmente distribuidas entre las especies, por tanto N0 es la riqueza, N1 es el número de las especies abundantes en la muestra, correspondiente al exponencial del índice de Shannon y N2 mide las especies muy abundantes, es equivalente al reciproco del Indicie de Simpson (Ludwing \& Reynolds, 1988). Como medida de equidad $\left(J^{\prime}\right)$ se usó la propuesta por Pielou, (1969) $\left(J^{\prime}=H^{\prime} / H_{\max }^{\prime}\right)$. Los valores de N0, $\mathrm{N} 1$, N2 y $J^{\prime}$ se calcularon en el programa MVSP versión 3.0 (Kovach, 1998).

Para conocer la validez del muestreo y la solidez de los índices de diversidad (Lampert \& Sommer, 1997), se utilizaron los estimadores de riqueza de especies Chao 1 y Chao 2, basados en las abundancias e incidencias, respectivamente (Colwell \& Coddington, 1994). Los valores de dichos estimadores se obtuvieron usando el programa EstimateS 6.0b1 (Colwell, 1997).

Para establecer significancia estadística de N0, N1, N2, $J^{\prime}$ y densidad, así como del alto de la columna de agua, el pH, el OD y la temperatura, entre estaciones y campañas de muestro, se utilizó un Análisis de Varianza (ANDEVA). En 
a)

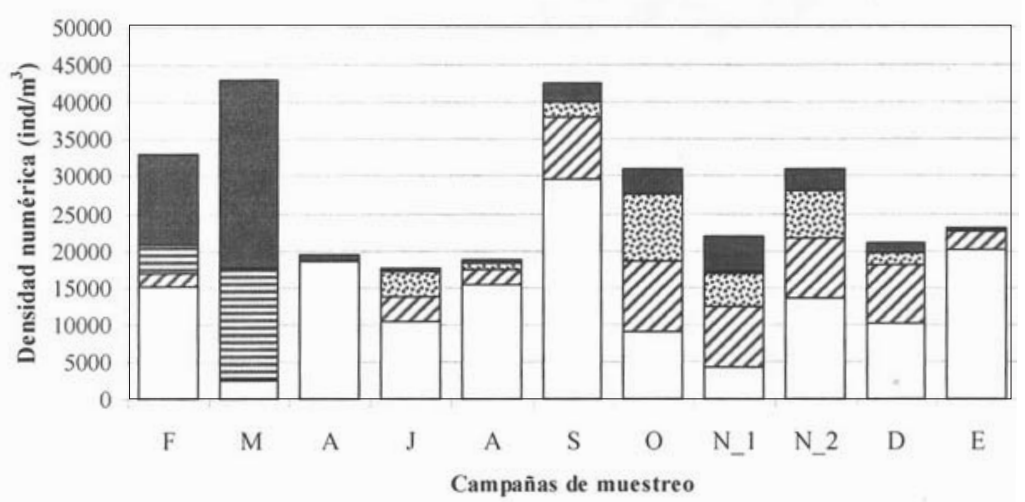

b)

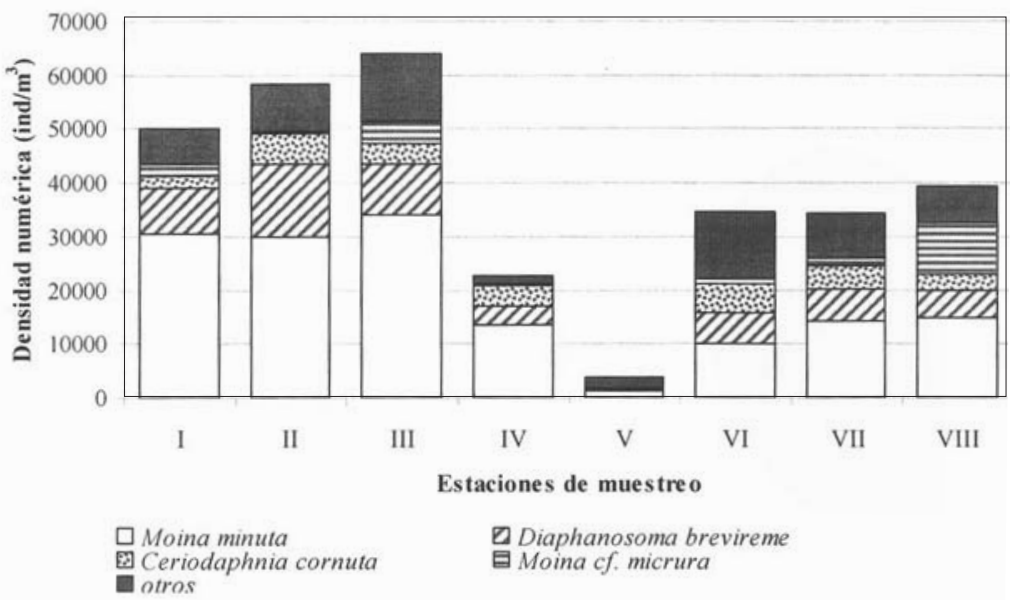

Figura 2. Variación de la densidad numérica total de Cladocera y de las especies más abundantes en: a) Campañas muestreo y b) Estaciones de muestreo. Variation of the total numeric density of Cladocera and the most abundant species in: a) Field trips, b) Sampling stations.

caso de obtener diferencias significativas, se utilizó la prueba a posteriori de Scheffé. Para estos análisis se empleo el programa Statistica v 5.1 (Statsoft, 1996).

\section{RESULTADOS}

Se encontraron 19 especies y 12 morfoespecies, pertenecientes a dos órdenes y siete familias (Tabla 2). Del total de las especies y las morfoespecies encontradas, las más abundantes represen- tan el $81.9 \%$ (Moina minuta, $49.2 \%$; Diaphanosoma brevireme, $17.2 \%$; Ceriodaphnia cornuta, $9.3 \%$ y Moina cf. micrura, $6.2 \%$ ); las especies y morfoespecies restantes representan el 18.1\%. La variación de la abundancia relativa de las especies y morfoespecies dominantes en las campañas y estaciones de muestreo, se puede observar en las figuras $2 \mathrm{a}$ y $2 \mathrm{~b}$, en las que se ve que el mayor porcentaje lo presenta M. minuta, con excepción de las campañas de muestreo de marzo, octubre y principios de noviembre y las 
estaciones $\mathrm{V}$ y VI en las que predominaron las especies D. brevireme, C. cornuta y M.cf. micrura.

Durante las campañas de muestreo los valores máximos densidad numérica se presentaron, en marzo y en septiembre, con valores de 5373 $\mathrm{ind} / \mathrm{m}^{3}$ y $5318 \mathrm{ind} / \mathrm{m}^{3}$ respectivamente, y valores mínimos en julio, agosto y diciembre (Fig. 3a). En cuanto a las estaciones de muestreo los cladóceros alcanzaron su máxima densidad numérica en la EIII, con $5822 \mathrm{ind} / \mathrm{m}^{3}$ y mínima en la estación $\mathrm{V}$ con $339 \mathrm{ind} / \mathrm{m}^{3}$ (Fig. 3b). El valor medio de la densidad fue $3491 \mathrm{ind} / \mathrm{m}^{3}$, con un máximo de $12636 \mathrm{ind} / \mathrm{m}^{3}$ y un mínimo de $22 \mathrm{ind} / \mathrm{m}^{3}$.

De las 31 especies y morfoespecies observa- das, los estimadores Chao 1 y 2 (Fig. 4a) infieren que no se incrementará este número en la ciénaga si se aumentara el esfuerzo de muestreo con las técnicas utilizadas. El comportamiento de los únicos, singletones, duplicados y dobletones (Fig. 4b) reveló que el muestreo fue suficiente (Colwell \& Coddington, 1994). Por lo tanto, la equidad y los números de Hill evaluados, están basados en muestreos efectivos.

Los evaluadores numéricos de estructura $\left(J^{\prime}\right.$, N0, N1, N2), la abundancia de cladóceros y el pH presentaron diferencias significativas entre estaciones de muestreo (Scheffé, $p=0.000$ a 0.001 ); dichas diferencias fueron entre la estación $\mathrm{V}$ y el

a)

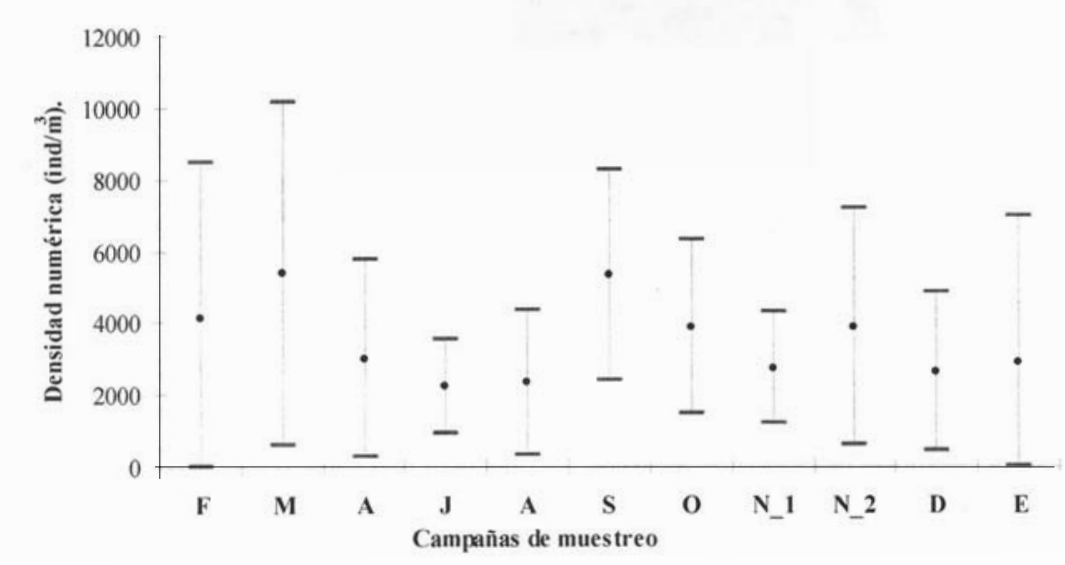

b)

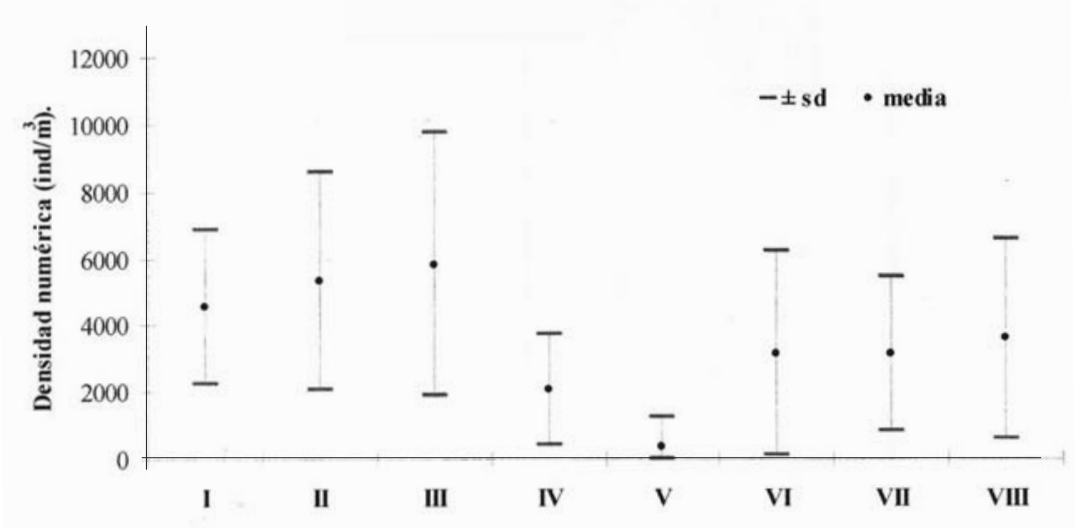

Estaciones de muestreo

Figura 3. Cambios de la densidad numérica de Cladocera en: a) Campañas muestreo y b) Estaciones de muestreo. Changes in the numeric density of Cladocera in: a) Field trips, b) Sampling stations. 
Tabla 2. Composición de Cladocera, en la Ciénaga de Paredes (Santander-Colombia). Composition of Cladocera in the Ciénaga de Paredes (Santander-Colombia).
ORDEN CTENOMOPODA FAMILIA SIDIDAE

ORDEN ANOMOPODA FAMILIA DAPHNIDAE

FAMILIA MOINIDAE

FAMILIA BOSMINIDAE

FAMILIA MACROTRICIDAE

FAMILIA ILYOCRYPTIDAE FAMILIA CHYDORIDAE SUBFAMILIA CHYDORINAE SUBFAMILIA ALONINAE
Diaphanosoma spinulosum Diaphanosoma dentatum Diaphanosoma brevireme Latonopsis sp Sarcilatona serricauda Pseudosida bidentata

Ceriodaphnia cornuta Simocephalus latirostris Scapholeberis armata Moina minuta Moina cf. micrura Moinodaphnia macleayii Bosmina hagmanni Bosmina tubicen Bosminopsis deitersi Macrothrix sp Grimaldina brazzai Ilyocryptus spinifer

Ephemeroporus tridentatus Dadaya macrops Leydigia cf. striata Alonella cf. dadayi Alonella sp

Kurzia latissima Camptocercus $\mathrm{sp}$ Notoalona $\mathrm{sp}$ Alona sp1 Alona sp2 Alona sp3 Alona sp4 Graptoleberis testudinaria

resto de estaciones (Scheffé, $p=0.000-0.034$ ). El alto de la columna de agua, el OD y la temperatura no presentaron diferencias significativas espaciales $(p=0.666$ a 0.999$)$. Entre campañas de muestreo se presentaron diferencias significativas en la equidad, la riqueza, en el alto de la columna de agua, el OD, la temperatura y el $\mathrm{pH}$, presentando diferencias entre campañas para las tres últimas (Scheffé, $p=0.000$ ), ya que para la equidad se encontraron diferencias entre las campañas de abril y finales de noviembre (Scheffé, $p=0.035$ ).

La riqueza temporal, presentó valores máximos en las campañas de abril y de enero y mínimos en la de diciembre (Fig. 5a). En cuanto a las estaciones, la mayor riqueza se encontró en la estación V y el menor en la estación VIII (Fig. $5 b)$. Los números de Hill N1 (especies abundantes) y N2 (especies muy abundantes), presentaron una tendencia similar a lo largo de las estaciones y campañas de muestreo (Figs. 6a y 6b), con valores máximos en las campañas de octubre y de finales de noviembre y mínimos en la campaña de agosto. Los valores máximos de N1 y N2 se presentaron en la estación V. Los mayores valores de equidad, se presentaron en la campaña de finales de noviembre $(0.71)$ y en la estación $\mathrm{V}(0.77)$ y los menores valores de equidad en la

a)

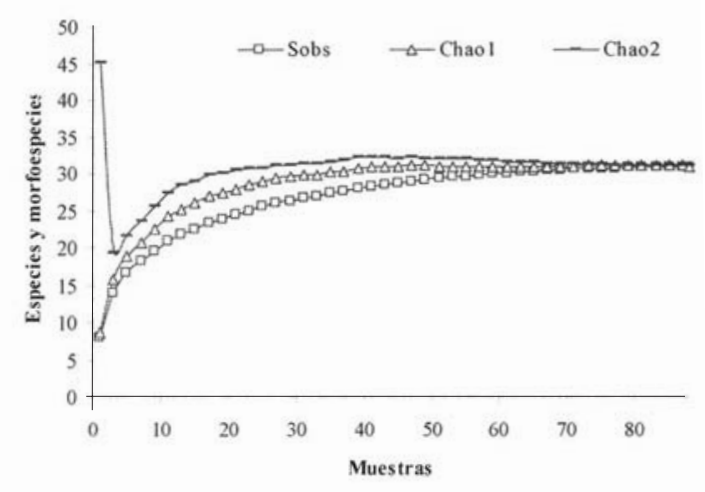

b)

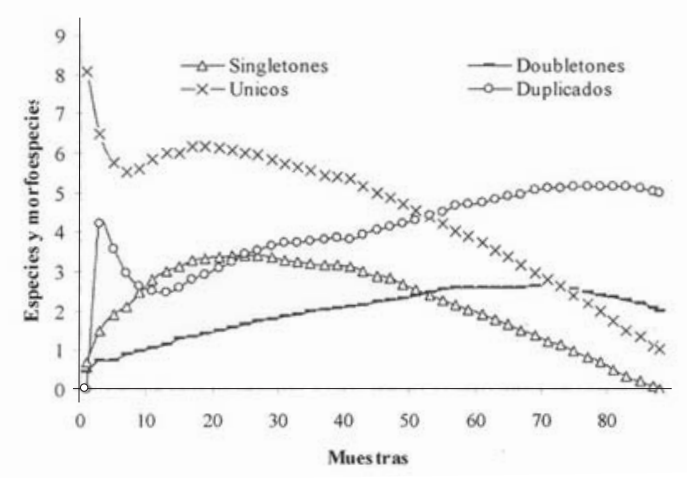

Figura 4. Curvas de acumulación de especies y morfoespecies: a) Estimadores Chao 1, Chao 2 y morfoespecies observadas (Sobs), b) únicos, duplicados, singletones y dobletones de Cladocera. Cummulative curves for species and morphospecies: a) Esimators Chao 1, Chao2, and observed morphospecies (Sobs), b) unique, duplicates, singletons and doubletons of Cladocera. 
campaña de abril (0.34) y en todas las estaciones a excepción de la estación V (0.57 a 0.45).

\section{DISCUSIÓN}

La composición de las especies y las morfoespecies encontradas en esta investigación es común en zonas tropicales. Resultados semejantes han sido registrados en Centro y Sur América (Hutchinson, 1967; Collado et al., 1984; Cisneros \& Manga, 1991; Infante, 1993; Ramírez \& Díaz, 1996-1997). Especies como Diaphanosoma spinulosum, D. brevireme, D. dentatum, Latonopsis sp, Sarcilatona serricauda, Pseudosida bidentata, Moina minuta, Moinodaphnia macleayii, Bosmina hagmanni, B. tubicen, Bosminopsis deitersi y Simocephalus latirostris presentan distribución tropical o subtropical (Goulden, 1968; Korovchinsky, 1992; Elmoor-Loureiro, 1998; Orlova Bienkowskaja, 2001; Kor̆inek, 2002). Moina micrura, Graptoleberis testudinaria, Kurzia latissima, Ilyocryptus spinifer son cosmopolitas (Oliver, 1960; Montú \& Goeden, 1986; ElmoorLoureiro, 1998; Hudec, 2000). De otro lado especies como M. minuta, C. cornuta y M. micrura, registradas en este trabajo han sido relacionadas por algunos autores como indicadoras de condiciones eutróficas u oligotróficas (Hutchinson, 1967; Pennak, 1978; Cisneros et al., 1991; Crispim \& Watanabe, 2000; Sampaio et al., 2002).

En lo que se refiere a la composición y al igual que en esta investigación Zambrano (1989) en la Laguna de Paca (3364 m de altitud), Estrada (1999) en el Embalse La Fé (2160 m.s.n.m) y Gavilán-Díaz et al. (2003) en La Laguna de Aguas Claras (3600 m.s.n.m.), encontraron especies de cladóceros diferentes a las halladas en menores altitudes como ha sido reportado por Crispim \& Watanabe (2000) y por Bohrer et al. (1988) para zonas subtropicales. Este patrón según Dumont (1994) ocurre porque hay sustitución de especies como Daphnia por especies más pequeñas en las altitudes bajas o porque con la altitud cambian las condiciones físicas y químicas de los cuerpos de agua, lo cual conlleva a diferencias en la composición de las especies entre lugares de altas y bajas altitudes (Lewis, 1987).
Las especies identificadas en este trabajo, $C$. cornuta, B. deitersi, B. hagmanni, M. minuta y $M$. cf. micrura, han sido encontradas como especies dominantes en cuerpos de agua temporales, en planos de inundación o en ciénagas, principalmente en la Amazonía Colombiana y Brasileña (Bozelli, 1994; Hardy et al., 1984; Sampaio et $a l ., 2002$ ), en la cuenca del Orinoco (Astiz \& Alvarez, 1998) y en algunos sistemas acuáticos tipo planos de inundación en Brasil y Venezuela (Zoppi de Roa et al., 1985; Pinto-Coelho, 1987; Rey \& Vásquez, 1988; Bozelli, 1992).

Los datos de diversidad obtenidos pueden ser comparados con escasos estudios de sistemas acuáticos ya que existen pocos trabajos con índices sólo para los cladóceros. Gavilán-Díaz (2000) obtuvo el índice de ShannonWiener para tres ciénagas del Magdalena Medio Santandereano. Al transformar estos valores a N1 (Chucurí 4.14, Llanito 2.93 y Paredes 4.62). El valor promedio de la presente investigación fue 3.43 , el cual se encuentra entre los valores de las ciénagas anteriormente mencionadas; por tanto la estructura de la comunidad de los cladóceros obtenida presenta según N1 un comportamiento similar al de otros cuerpos de agua cenagosos de la misma región.

La riqueza de cladóceros se encuentra entre las esperadas (30 y 50 taxones) para sistemas acuáticos tropicales (Dumont, 1994), así como para algunos planos de inundación de Africa (Dumont et al., 1981), para aguas lóticas del Paraná medio (Paggi \& Paggi, 1974) y para la Lagoa dos Patos en Brasil (Montú \& Goeden, 1986). Sin embargo los datos encontrados por Astiz \& Alvarez (1998) para el alto y medio Orinoco, por Hardy et al. (1984) para Lago Camaleão y por Crispim \& Watanabe (2000) para algunos planos de inundación de Brasil, son menores (10 y 26 taxones).

La estación $\mathrm{V}$ presentó una tendencia atípica en comparación con las otras estaciones, como lo demostraron el análisis de varianza y la prueba $a$ posteriori; estos resultados son explicados por la cercanía de la estación $\mathrm{V}$ al afluente principal de la ciénaga (Quebrada La Gómez), estación que presentó menor densidad numérica y mayor diversidad (N1 y N2), equidad ( $\left.J^{\prime}\right)$ y riqueza (N0). 

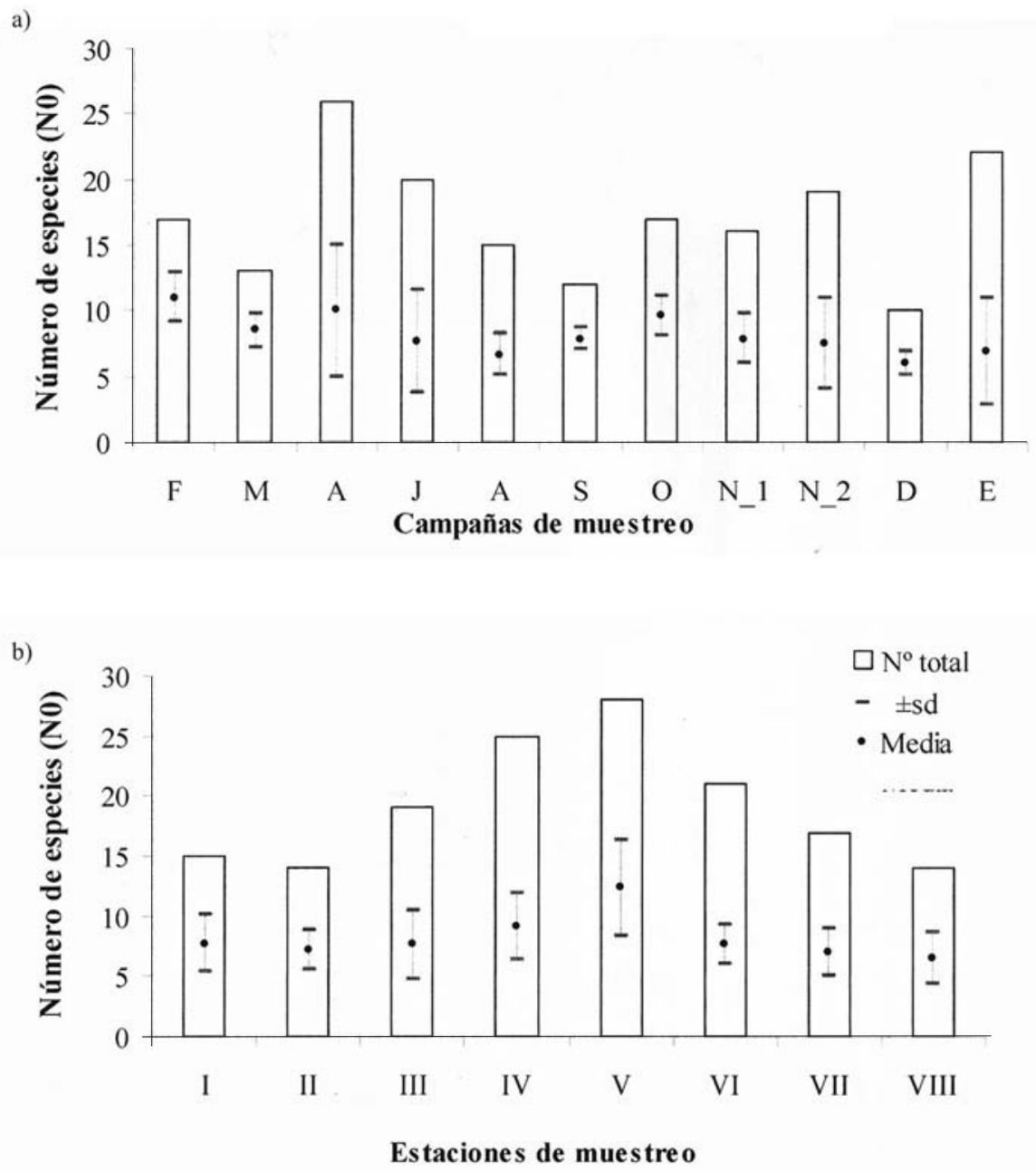

Figura 5. Dinámica de la riqueza de Cladocera en un ciclo anual en la Ciénaga de Paredes en: a) Campañas de muestreo, b) Estaciones de muestro. Cladocera richness dynamics in an annual cycle in the Ciénaga de Paredes in: a) Field trips, b) Sampling stations.

Hecho que no puede ser atribuido a la variación de la columna del agua ya que esta no presentó diferencias significativas entre las estaciones de muestreo, ni a la presencia de macrófitas flotantes ya que estas se presentaron en las tres últimas campañas de muestreo en toda la ciénaga.

La tendencia atípica de la estructura de la comunidad de los cladóceros en la estación $\mathrm{V}$ es atribuido según lo encontrado en este estudio a: 1) las diferencias de $\mathrm{pH}$ entre la estación $\mathrm{V}$ y el resto de las estaciones, ya que el $\mathrm{pH}$ es una variable para la cual las poblaciones de cladóceros presentan estrechos niveles de tolerancia para su desarrollo (Lampert \& Sommer, 1997); y 2) a que lugares expuestos a perturbaciones frecuentes y de magnitud moderada Connell (1978), en este caso el movimiento y la fluctuación de nutrientes debidas a las corrientes, presentan mayor diversidad. Thompson \& Townsend (2000), lo explican diciendo que las perturbaciones de las comunidades en arroyos pueden deberse a variación en el caudal. Adicionalmente es importante resaltar lo encontrado por Montenegro (1995) quien estudio varias estaciones de muestreo entre las que se encontraban lugares cercanos a la desembocadura de la Quebrada La Gómez (estación 
a)

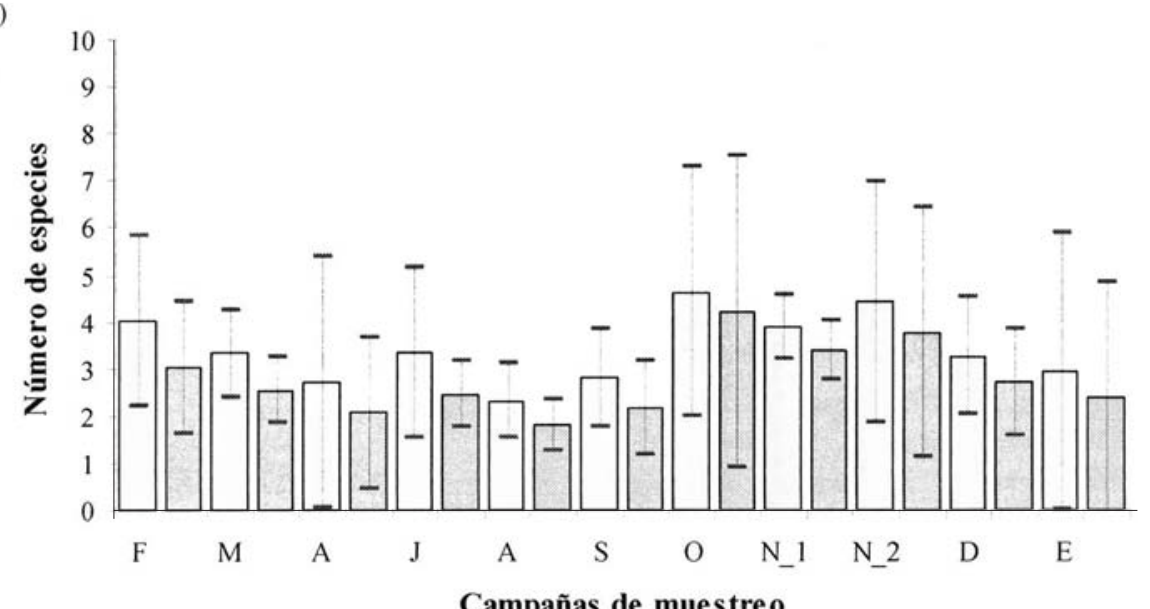

Campañas de muestreo

b)

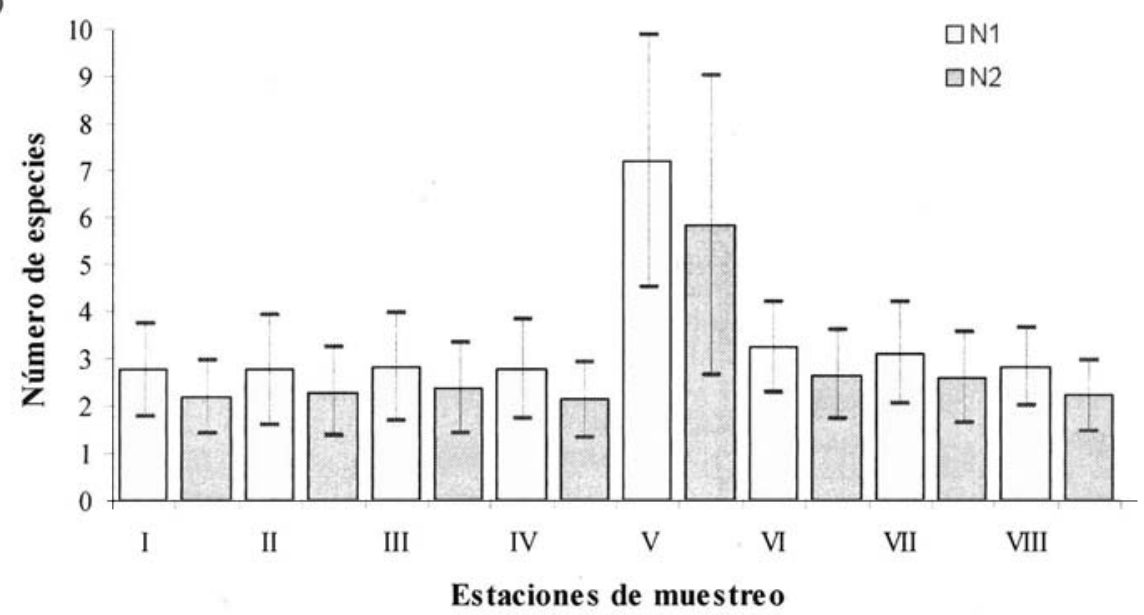

Figura 6. Variación de los números de Hill N1 y N2 en: a) Campañas muestreo, b) Estaciones de muestro. Variation of the Hill Numbers N1 and N2 in: a) Field trips, b) Sampling stations.

V); esta estación se caracterizó por la presencia de macrófitas sumergidas, menor densidad fitoplanctónica y menor densidad de cladóceros y copépodos, lo cual concuerda con lo hallado en esta investigación e indica la existencia de poco alimento disponible en este lugar.

Adicionalmente, las diferencias en la abundancia de cladóceros entre estaciones indican que las condiciones en la estación $\mathrm{V}$ no favorecen densidades mayores a las halladas en este trabajo. Barón-Rodríguez et al. (2005), al aplicar un PCA en esta Ciénaga en el mismo periodo de estudio encontraron que para todos los puntos de muestreo excepto para la estación $\mathrm{V}$, la abundancia de cladóceros estuvo relacionada positivamente con el $\mathrm{pH}$, la turbidez, la temperatura del agua y la heterogeneidad de la columna para estas variables, mientras que la relación con la transparencia fue negativa. La referencia anterior verifica los resultados del análisis en este ítem.

La variación temporal de la estructura se refleja en las diferencias significativas encontradas en la equidad y la riqueza entre las campañas de muestreo a través del ciclo. El hecho de no encontrar las mismas especies y morfoespecies dominantes indica que temporalmente hubo sustitución de especies y por lo tanto cambio en sus respectivas densidades numéricas. Estos resultados son consecuencia de los cambios temporales en las condiciones físicas y químicas 
del sistema debidas al régimen climático. Barón-Rodríguez et al. (2005), describen la relación positiva entre la precipitación, el nivel del agua y la transparencia con las variables bióticas equidad y diversidad, y la relación negativa entre éstas y la turbidez, los nutrientes, el $\mathrm{pH}$, la conductividad y la densidad numérica. Este análisis permite establecer que existen las condiciones adecuadas para el desarrollo y temporalidad de la comunidad de cladóceros.

Se concluye entonces que en cuanto a la variación espacial de la estructura la estación $\mathrm{V}$ presentó condiciones que ocasionaron una disminución en la densidad numérica, aumento de la equidad, la riqueza y la diversidad, contexto que es explicado por las características bióticas y abióticas del lugar y por la influencia de perturbación de carácter intermedio. La variación temporal de la estructura de la comunidad de cladóceros se debió a las fluctuaciones en la equidad, la riqueza y el cambio de la abundancia relativa de las morfoespecies; lo anterior, debido a la influencia de la variación de la precipitación y de la columna de agua en el sistema acuático en estudio. En general la estructura de la comunidad de cladóceros presente en la ciénaga de Paredes mostró heterogeneidad espacial y temporal.

\section{AGRADECIMIENTOS}

Los autores agradecen a las profesoras Lourdes María Elmoor Loureiro (Universidade Católica de Brasilia), Odete Rocha (Universidad Federal de São Carlos -Brasil), Evelyn Zoppi de Roa (Universidad Central de Venezuela) y Marina Manca (Istituto per lo Studio degli EcosistemiCNR), por confirmar las especies encontradas.

\section{BIBLIOGRAFÍA}

AMERICAN PUBLIC HEALTH ASSOCIATION. (APHA). 1995. Standard Methods for the Examination of Water and Wastewater. $19^{\text {th }}$ ed. Washington. $874 \mathrm{pp}$.

ARIAS, P. 1985. Las Ciénagas de Colombia. Revista Divulg. Pesq. Inderena, 22: 39-70.
ASTIZ, S. \& H. ÁLVAREZ. 1998. El zooplancton en el alto y medio Río Orinoco, Venezuela. Acta Científica Venezolana, 49: 5-18.

BARON-RODRÍGUEZ M. M., R. A. GAVILÁNDÍAZ \& J. J. RAMÍREZ. 2005. Factor and variables related to the temporl and spatial fluctuation of the numeric density of Cladocera in a lowland neotropical ciénaga. VII ${ }^{\text {th }}$ International Symposium on Cladocera. September 3-9, 2005, Herzberg. Switzerland.

BOHRER, M. B. C., M. M. ROCHA \& B. F. GODOLPHIM. 1988. Variações espaço-temporais das populações de Cladocera (Crustacea-Branchiopoda) no Saco de Tapes, Laguna Dos Patos, R.S. Acta Limnol. Brasil., 11: 549-570.

BOZELLI, R. L. 1992. Composition of the zooplankton community of Batata and Mussurá Lakes and of the Trombetas River, State of Pará, Brazil. Amazoniana, XII (2): 239-261.

BOZELLI, R. L. 1994. Zooplankton community density in relation to water level fluctuations and inorganic turbity in an Amazonian lake, "Lago Batata", State of Pará, Brazil. Amazoniana, XIII(1/2): 17-32.

BROOKS, J. L. 1959. Cladocera. In: Fresh-Water Biology. W. T. Edmonson (ed): 587-656. $2^{\text {ond }}$ ed. John Willey \& Sons, INC. USA.

CISNEROS, R. \& E. I. MANGA. 1991. Zooplankton studies in a tropical lake (Lake Xolotlán, Nicaragua). Verh. Internat. Verein. Limnol., 24: 11671170.

CISNEROS, R., E. I. MANGA \& M. VAN MAREN. 1991. Qualitative and quantitative structure, diversity and fluctuations in abundance of zooplankton in a lake Xolotlán (Managua). Hydrobiol. Bull., 25(2): 151-156.

COLLADO, C., C. H. FERNANDO \& D. SEPHTON. 1984. The freshwater zooplankton of Central America and the Caribbean. Hidrobiologia, 113: 105-119.

COLWELL, R. K., \& J. A. CODDINGTON. 1994. Estimating terrestrial biodiversity through extrapolation. Phil. Trans. Royal Soc., (Series B) 345: 101118.

COLWELL, R. K. 1997. EstimateS: statistical estimation of species richness and shared species from samples. Version $6.01 \mathrm{~b}$. User's Guide and application published at: http://viceroy.eeb.uconn.edu/ estimates

CONNELL, J. H. 1978. Diversity in tropical rain forest and coral reefs. Science, 199: 1302-1310. 
CORMAGDALENA, 1999. Río Magdalena. Visita técnica Ciénagas de Paredes, El Llanito y Chucurí (Santander). IGAC. 12 pp.

CRISPIM, M. C. \& T. WATANABE. 2000. Caracterização Limnológica das bacias doadoras o receptoras de águas do Rio São Francisco: 1-Zooplancton. Acta. Limnol. Bras., 12: 93-103.

DUMONT, H. J., J. PENSAERT \& I. V. DE VELDE. 1981. The crustacean zooplankton of Mali (West Africa). Hydrobiologia, 80: 161-187.

DUMONT, H. J. 1994. On the diversity of the Cladocera in the tropics. Hydrobiologia, 272: 29-38.

ELMOOR-LOUREIRO, L. M. A. 1997. Manual de identificaçâo de Cladóceros límnicos do Brasil. Editora Universa. Universidad Católica de Brasilia. Brasil. 155 pp.

ELMOOR-LOUREIRO, L. M. A. 1998. Branchiopoda Freshwater Cladocera. In: Cataloque of Crustacea of Brazil. P. S. Young (ed.): 15-41. Rio de Janeiro: Museu Nacional.

ESTRADA, A. L. 1999. Variação espacial e temporal da comunidade zooplanctónica do reservatório La Fé, Antioquia, Colombia. Tese grado, Universidade de São Paulo. 130 pp.

FERNANDO, C. H., C. TUDORANCEA \& S. MENGESTOU. 1990. Invertebrate zooplankton predator composition and diversity in tropical lentic waters. Hidrobiologia, 198: 13-31.

FLÖSSNER, D. 2000. Die Haplopoda und Cladocera (ohne Bosminidae) Mitteleuropas. Backhuys Publishers, Leiden, The Netherlands. 428 pp.

GARCÍA, L. \& E. DISTER. 1990. La planicie de inundación del medio-bajo magdalena: restauración y conservación de hábitats. Interciencia, 15: 396-410.

GAVILÁN-DÍAZ, R. A. 2000. Análisis de la diversidad en ciénagas del Magdalena Medio Santandereano (Neotrópico) con énfasis en la Comunidad zooplanctónica y el ciclo hidrológico regional. Fase I y II. Informe Convenio UIS-Cormagdalena. $353 \mathrm{pp}$.

GAVILÁN-DÍAZ, R. A., Y. PLATA-DÍAZ \& M. M. BARÓN-RODRÍGUEZ. 2003. Plancton de un lago de Alta Montaña neotropical (Aguas ClarasSantander-Colombia). Informe Final del contrato de investigación 319-2000. Colciencias -UISGrupo de Estudios en Biodiversidad. 58-84 pp.

GOULDEN, C. E. 1968. The systematics and evolution of the Moinidae. Trans. Am. Phil. Soc., 58: 1101.
HANEY, J. F. \& D. J. HALL. 1973. Sugar-coated Daphnia: a preservation technique for Cladocera. Limnol. Oceanogr., 18: 331-339.

HARDY, E. R., B. ROBERTSON \& W. KOSTE. 1984. About the relationship between the zooplankton and fluctuating water levels of Lago Camaleão, a Central Amazonian vãrzea lake. Amazoniana, IX: 43-52.

HILL, N. O. 1973. Diversity and evenness: A unifying notation and its consequences. Ecology, 54: 427-432.

HOLDRIDGE, R. 2000. Ecología basada en zonas de vida. Instituto Interamericano de Cooperación para la Agricultura. IICA, San José de Costa Rica. $216 \mathrm{pp}$.

HUDEC, I. 2000. Subgeneric differentiation within Kurzia (Crustacea: Anomopoda: Chydoridae) and a new species from Central America. Hydrobiologia, 421: 165-178.

HUTCHINSON, G. E. 1967. A treatise of Limnology. John Willey \& Sons, INC. New York. 1115 pp.

INFANTE, A. G. 1993. Vertical and horizontal distribution of the zooplankton in a lake Valencia. Acta. Limnol. Bras., VI: 97-105.

JUNK, W., P. B. BAYLEY \& R. E. SPARKS. 1989. The flood pulse concept in river-floodplain systems. Can. Spec. Publ. Fish. Aquat. Sci., 106: 110127.

JUNK, W. J. 1996. Ecology of floodplains -a challenge for tropical limnology. In: Perspectives in tropical limnology. F. Shiemer \& K. T. Boland (eds.): 255-265. SPB Academic Publishing, Amsderdam.

KEMPTON, R. A. 1979. The structure of species abundance and measurement of diversity. Biometrics, 35:307-321.

KOÏINEK, V. 2002. Cladocera. In: A Guide to Tropical Freshwater Zooplankton. C. H. Fernando (ed.): 69-122. Backhuys Publishers, Leiden, The Netherlands.

KOROVCHINSKY, N. M. 1992. Guides to the Identification of the Microinvertebrates of the Continental Waters of the World $N^{\circ} 3$. Sididae \& Holopodidae. (Crustacea: Daphniiformes). SPB Academic Publishing, The Netherlands. 86 pp.

KOROVCHINSKY, N. M. \& M. ELÍAS-GUTIÉRREZ. 2000. First record of Sarcilatona serricauda (Sars, 1901) (Crustacea: Branchiopoda: Sididae) from Mexico, with redescription of its male. Arthropoda Selecta., 9(1): 5-11. 
KOVACH, W. 1998. MVSP v 3.00b for Windows. Multivariate Statistical Package. UK.

LAMPERT, W. \& U. SOMMER 1997. Limnoecology. The ecology of lakes and streams. Oxford University Press. New York. 382 pp.

LANSAC TÔHA, F. A., A. F. LIMA, S. M. THOMAZ \& M. C. ROBERTO. 1993. Zooplancton de uma planicie de inundação do Rio Paraná. II. Variação sazonal e influência dos níveis fluviométricos sobre a comunidade. Acta Limnologica Braziliensia, (VI): 42-55.

LEWIS, W. 1987. Tropical Limnology. Ann. Rev. Ecol. Syst., 18: 159-184.

LIEDER, V. U. 1996. Crustácea. Cladocera/Bosminidae. Gstav Fischer, Berlin. 84 pp.

LUDWING, J. A. \& REYNOLDS, J. F. 1988. Statistical ecology. A primer on methods and computing. John Wiley \& Sons. New Cork. 337 pp.

MARGALEF, R. 1991. Ecología. Ed. Omega. Barcelona. $951 \mathrm{pp}$.

MONTENEGRO, M. I. 1995. Evaluación ambiental de la Ciénaga de Paredes. Sabana de Torres, Santander, como hábitat para fauna silverstre; con especial énfasis en el Manatí (Trichechus manatus) -Primera fase. Informe División de Fauna Terrestre- INDERENA \& Fundación para la Promoción de la Investigación y la Tecnología Banco de la República. 81 pp.

MONTÚ, M. \& I. M. GOEDEN. 1986. Atlas dos Cladocera e Copepoda (Crustacea) do Estuario da Lagoa Dos Patos (Rio Grande, Brasil). Neritica, 1(2): 1-134.

OLIVER, S. 1969. Los Cladóceros Argentinos con claves de las especies, notas biológicas y distribución geográfica. Revista del Museo de la Plata, 7: 173-269.

ORLOVA-BIENKOWSKAJA, M. Y. 2001. Guides to the Identification of the Microinvertebrates of the Continental Waters of the World $N^{\circ} 17$. Cladocera: Anomopoda. Daphniidae: genus Simocephalus. Backhuys Publishers, Leiden. The Netherlands. 128 pp.

PAGGI, C. P. \& S. J. PAGGI. 1974. Primeros estudios sobre el zooplancton de aguas lóticas del Paraná medio. Physis, 33(86): 91-114.

PAGGI, J. C. 1995. Crustácea Cladocera. En: Ecosistemas de Aguas Continentales. E. C. Lopretto y G. Tell (eds): 909-951. Tomo III. Ediciones Sur. Argentina.

PENNAK, R. W. 1978. Fresh-Water Invertebrates of the United States. Wiley. USA. 798 pp.
PIELOU, E. C. 1969. Ecological Diversity and Its Measurement. In: An Introduction to Mathematical Ecology. E. C. Pielou (ed.): 221-235. John Wiley \& Sons. United States of América.

PINTO-COELHO, R. M. 1987. Fluctuações sazonais e de curto duração na comunidade zooplanctónica do Lago Paranoá, Brasilia-D.F. Brasil. Rev. Brasil. Biol., 47: 17-29.

RAMÍREZ. J. J. \& A. DÍAZ. 1996-1997. Fluctuación estacional del zooplancton en la laguna del Parque Norte, Medellín, Colombia. Rev. Biol. Trop., 44/45: 549-563.

REY, J. \& E. VÁSQUEZ. 1988. Notas sobre los avances de las investigaciones de los cladóceros (Crustacea, Cladocera) de la Cuenca baja del Orinoco. Sociedad de ciencias Naturales La Salle, XLVIII: 155-161.

REY, J. 1991. Los Cladóceros. En: El Lago Titicaca: síntesis del conocimiento limnológico actual. Dejoux C. y A. Iltis (eds.): 265-276. ORSTOM. Bolivia.

ROESSLER, E. W. 1994. Los cladóceros de la familia Sididae Baird, 1850, en Colombia. (Crustacea, Cladocera). Comité de investigaciones, Universidad de los Andes, Bogotá, D. E., Colombia. 27 pp.

ROESSLER, E. W. 1996. La familia Moinidae en Colombia. (Arthropoda, Crustácea, Cladocera). Los Moinidae de la amazonía colombiana. Comité de investigaciones, Universidad de los Andes, Bogotá, D. E., Colombia. 25 pp.

SAMPAIO, E., O. ROCHA, T. MATSUMURATUNDISI \& J. G.TUNDISI. 2002. Composition and abundance of zooplankton in the limnetic zone of seven reservoirs of the Paranapanema River, Brazil. Braz. J. Biol., 62: 525-545.

SENDACZ. S., E. KUBO \& L. P. FUJIARA. 1984. Further studies on the zooplankton community of a eutrophic reservoir in southern Brazil. Verh. Internt. Verein. Limnol., 22: 1625-1630.

SENDACZ, S. \& E. KUBO. 1999. Zooplancton de reservatórios do Alto Tietê. In: Ecologia de reservatórios: estructura, função e aspectos sociais. R. Henry (ed.): 509-530. Botucatu, FUNDIOBIO/FAPESP.

SMIRNOV, N. N. 1992. Guides to the Identification of the Microinvertebrates of the Continental Waters of the World $N^{\circ}$ 1. The Macrothricidae of the World. SPB Academic Publishing, The Netherlands. $150 \mathrm{pp}$. 
SMIRNOV, N. N. 1996. Guides to the Identification of the Microinvertebrates of the Continental Waters of the World $N^{\circ}$ 11. Cladocera: the Chydorinae and Sayciinae (Chydoridae) of the World. SPB Academic Publishing, The Netherlands. 248 pp.

STATSOFT, INC., 1996. Statistica v 5.1 for Windows [Computer program manual]. Tulsa.

THOMPSON, R. M. \& C. M. TOWNSEND. 2000. New Zealand's Stream Invertebrate Communities: An International Perspective. In: New Zealand stream invertebrates: ecology and implications for management. K. J. Collier \& M. J. Winterbourn (eds.): 53-74. New Zealand Limnological Society, Christchurch.
VALDIVIA, R. 1988. Lista de Cladóceros dulceacuícolas del Perú. Amazoniana, X(3): 283-297.

WETZEL, R. G. \& G. E. LIKENS. 1990. Limnological Analyses. W. B. Saunders Company. USA. 391 pp.

ZAMBRANO, F. 1989. Estudio taxonómico de los cladóceros de la Laguna de Paca (Jauja-Junín). Tesis de Grado. Facultad de Ciencias Biológicas. Universidad Ricardo Palma. 141 pp.

ZOPPI DE ROA, E., F. MICHELANGELLI. \& L. SEGOVIA. 1985. Cladocera (Crustácea, Branchiopoda) de sabanas inundables de Mancal, Estado Apure, Venezuela. Acta Biol. Venez., 12: 4355. 